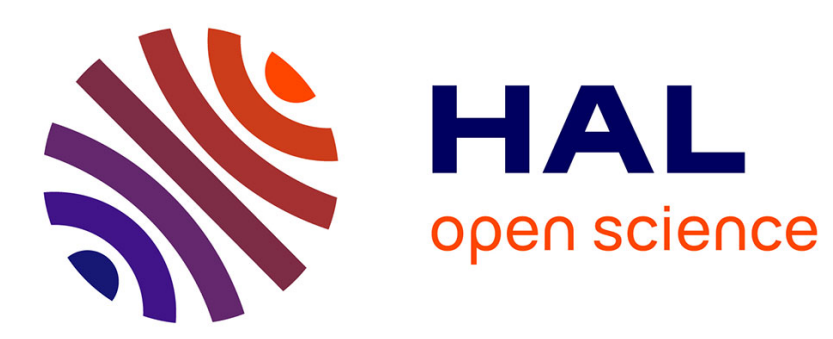

\title{
Simulation of the structure of organosilane film coatings
}

\author{
David James Willock, Graeme W. Watson, Robert A Hayes
}

\section{To cite this version:}

David James Willock, Graeme W. Watson, Robert A Hayes. Simulation of the structure of organosilane film coatings. Molecular Simulation, 2007, 32 (12-13), pp.1095-1101. 10.1080/08927020601052898 . hal-00515007

\section{HAL Id: hal-00515007 \\ https://hal.science/hal-00515007}

Submitted on 4 Sep 2010

HAL is a multi-disciplinary open access archive for the deposit and dissemination of scientific research documents, whether they are published or not. The documents may come from teaching and research institutions in France or abroad, or from public or private research centers.
L'archive ouverte pluridisciplinaire HAL, est destinée au dépôt et à la diffusion de documents scientifiques de niveau recherche, publiés ou non, émanant des établissements d'enseignement et de recherche français ou étrangers, des laboratoires publics ou privés. 


\section{Molecular Simulation}

Journal of

Experimental Nanoscience

- Taylor \& Francis

Taglor \& Francis Group

\section{Simulation of the structure of organosilane film coatings}

\begin{tabular}{|r|l|}
\hline Journal: & Molecular Simulation/Journal of Experimental Nanoscience \\
\hline Manuscript ID: & GMOS-2006-0128.R1 \\
\hline Journal: & Molecular Simulation \\
\hline $\begin{array}{r}\text { Date Submitted by the } \\
\text { Author: }\end{array}$ & $28-S e p-2006$ \\
\hline Complete List of Authors: & $\begin{array}{l}\text { Willock, David; Cardiff University, School of Chemistry } \\
\text { Watson, Graeme; Trinity College Dublin, School of Chemistry } \\
\text { Hayes, Robert; Cardiff University, School of Chemistry }\end{array}$ \\
\hline Keywords: & organosilane, coatings, molecular dynamics, moment of inertia \\
\hline
\end{tabular}

\section{SCHOLARONE ${ }^{\mathrm{m}}$ \\ Manuscripts}




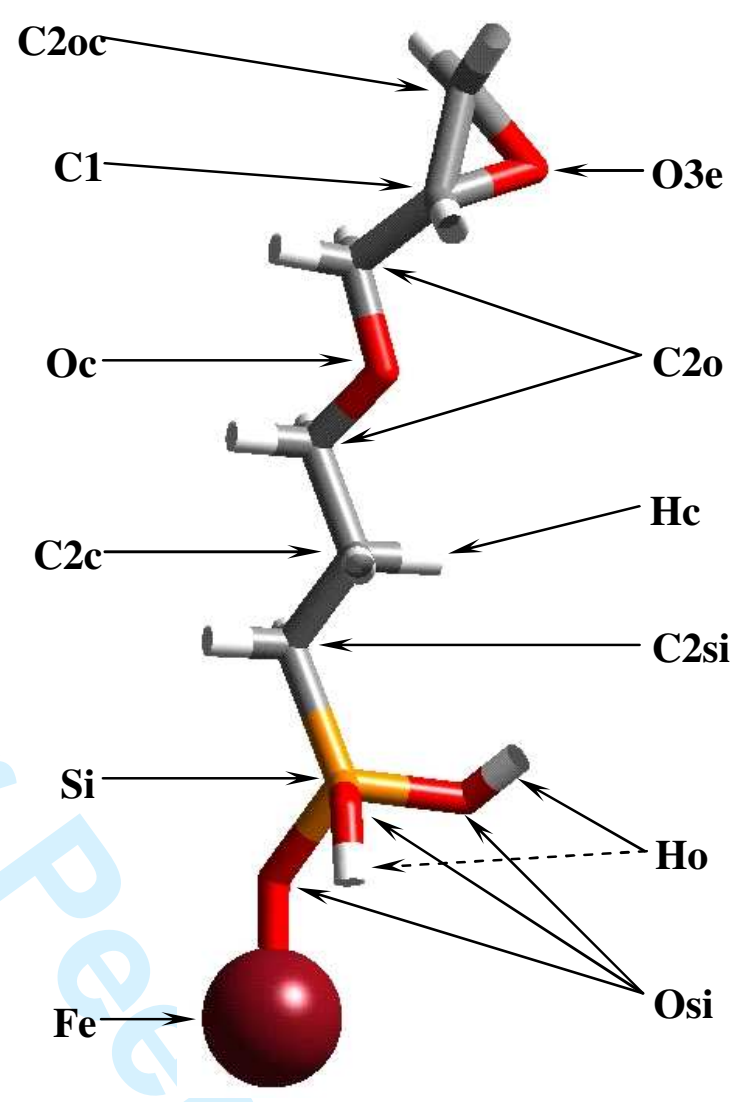


a)

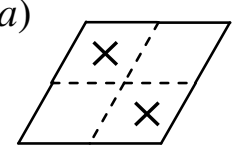

b)

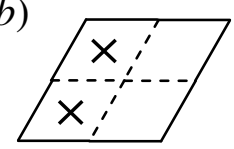




\section{Page 3 of 21}

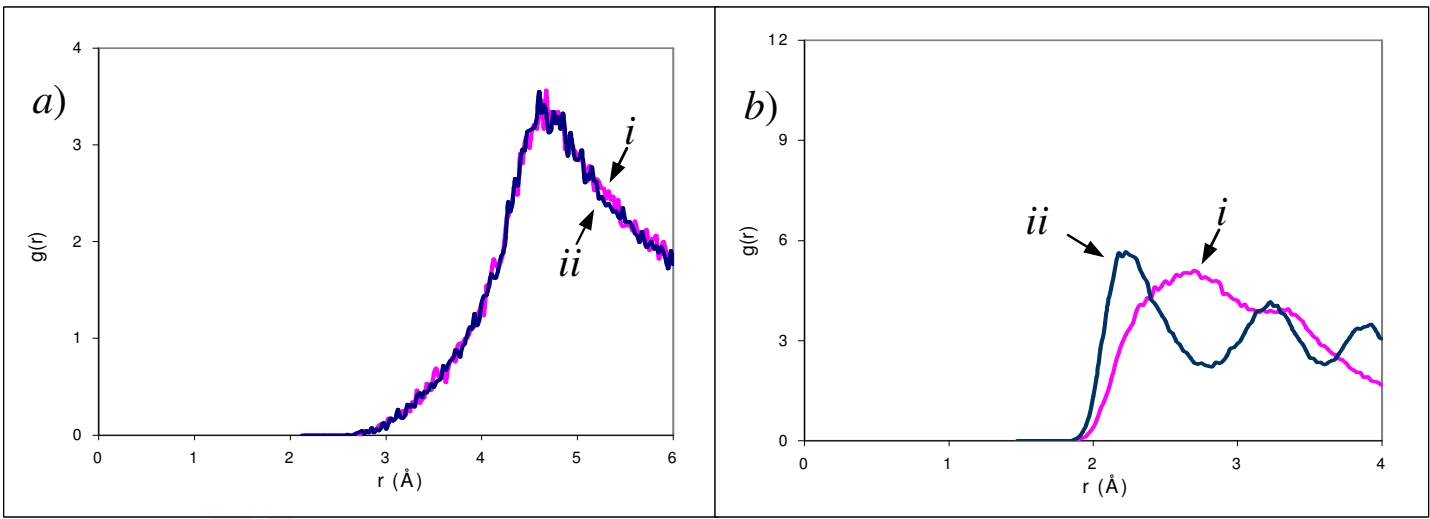

1

3

5

6

7

8

10

11

12

13

14

15

16

17

18

19

20

21

22

23

24

25

26

27

28

29

30

31

32

33

34

35

36

37

38

39

40

41

42

43

44

45

46

47

48

49

50

51

52

53

54

55

56

57

58

59

60 

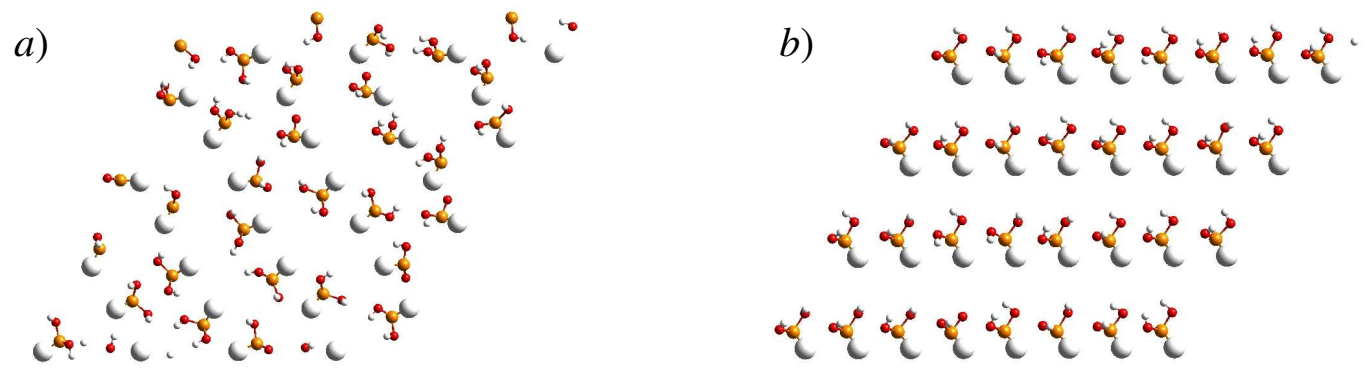


\section{Page 5 of 21}

1
2
3
4
5
6
7
8
9
10
11
12
13
14
15
16
17
18
19
20
21
22
23
24
25
26
27
28
29
30
31
32
33
34
35
36
37
38
39
40
41
42
43
44
45
46
47
48
49
50
51
52
53
54
55
56
57
58
59
60

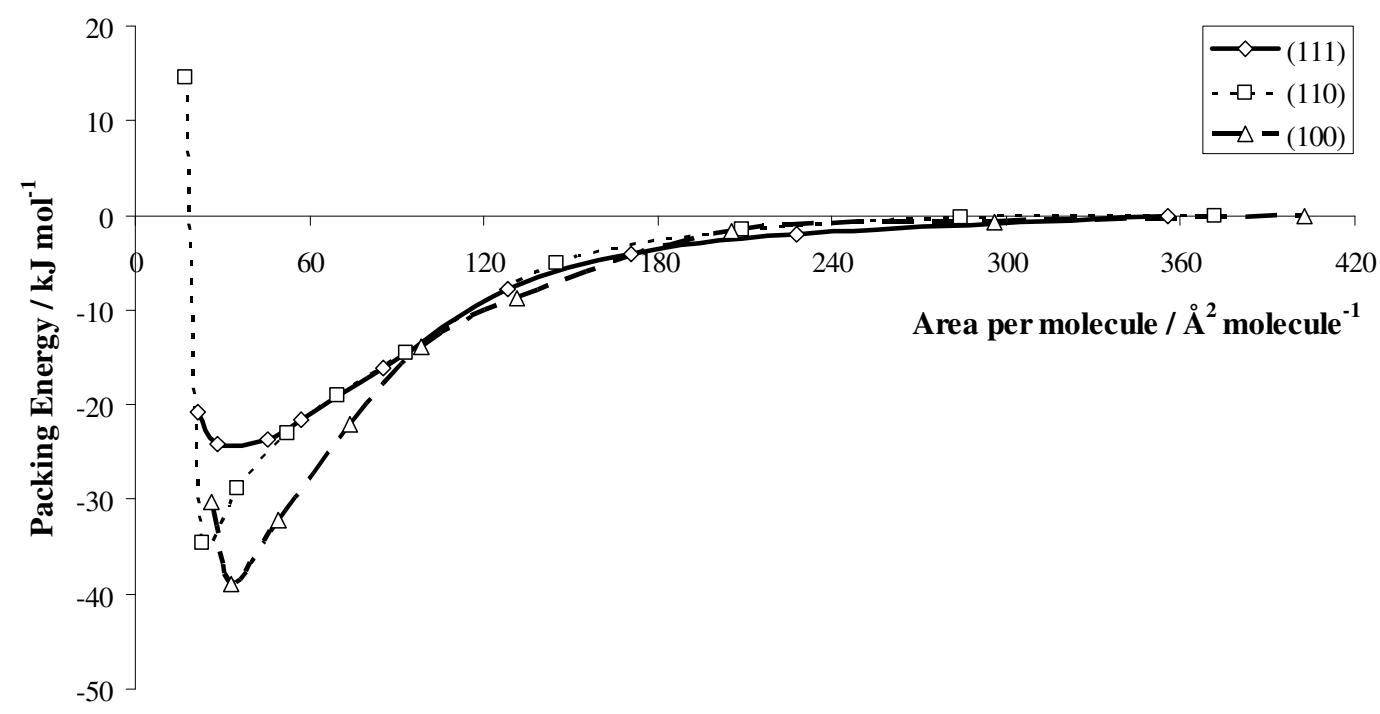




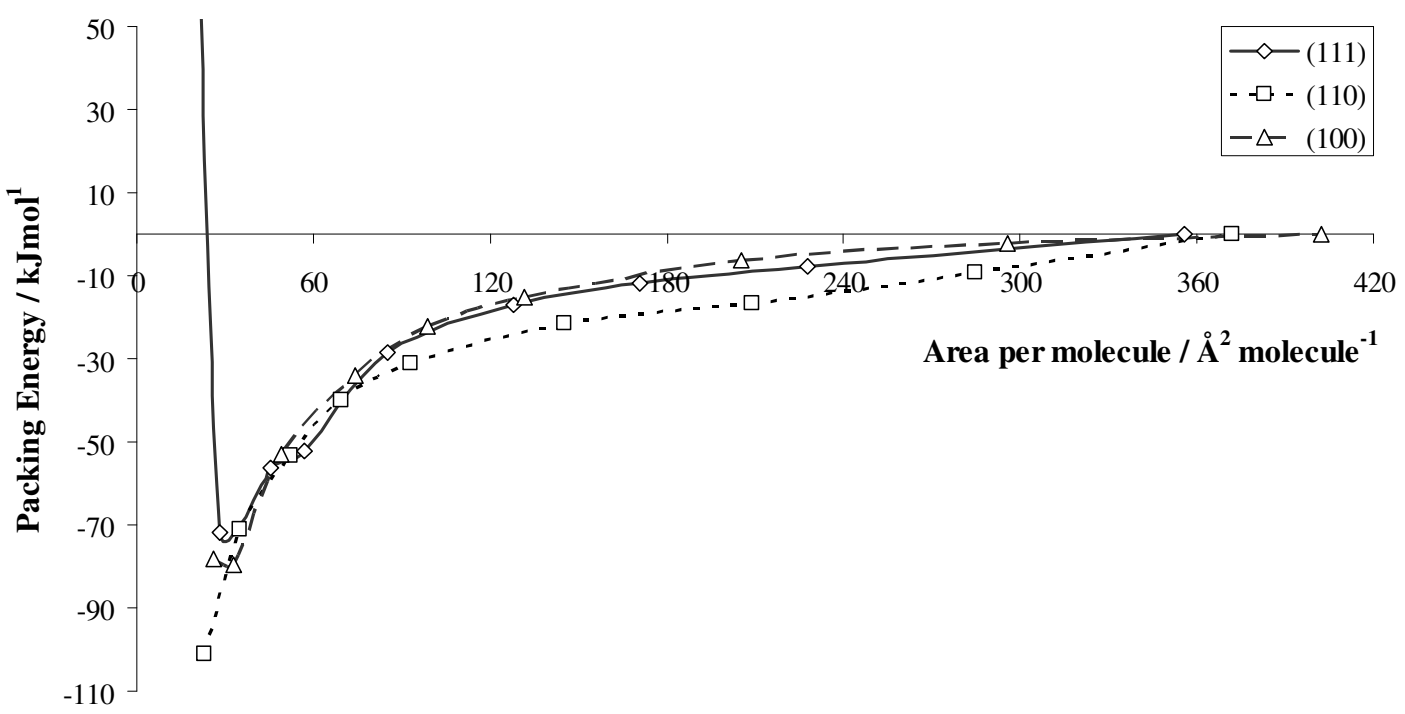




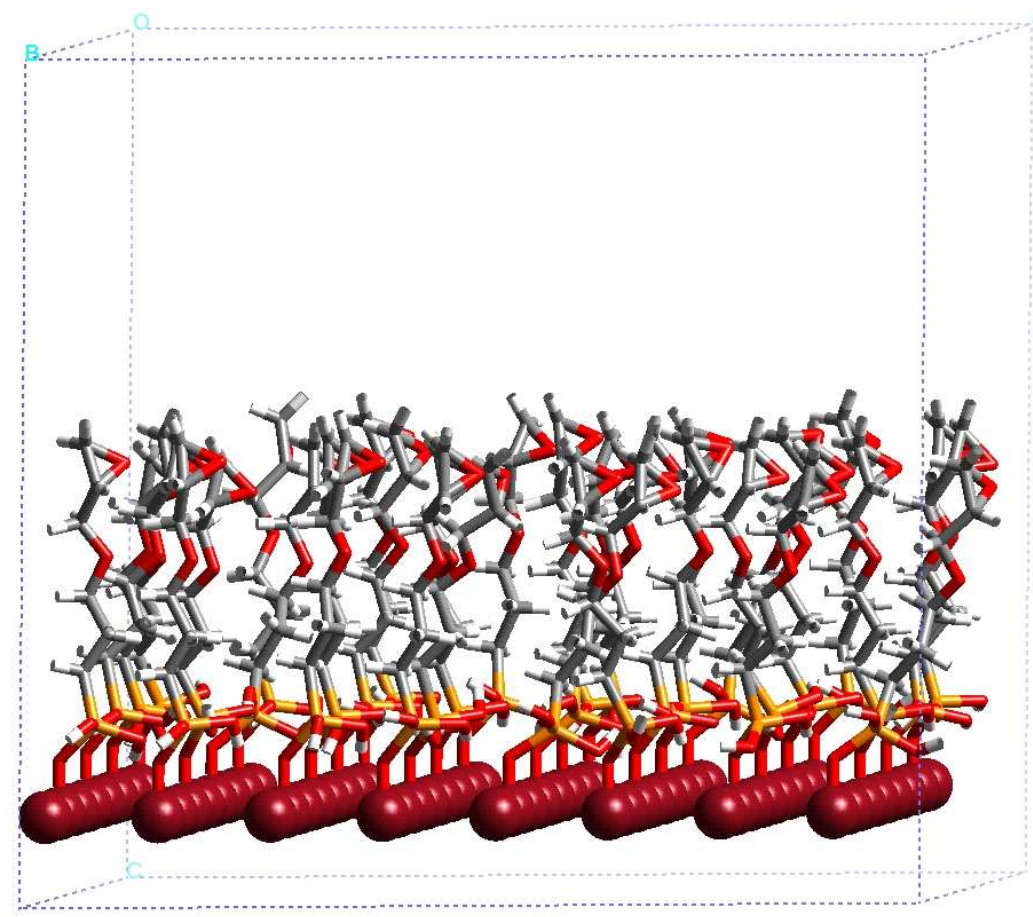



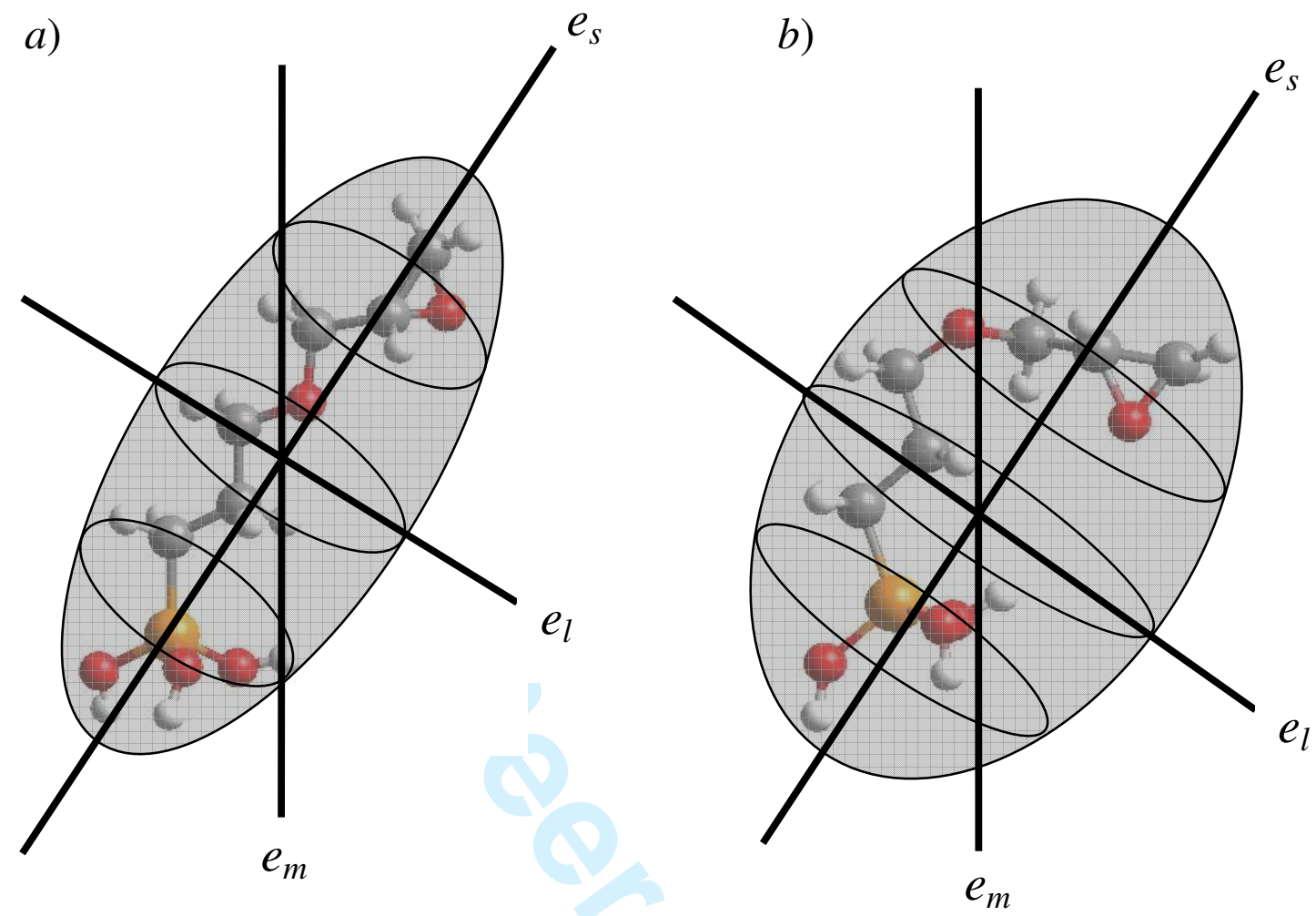

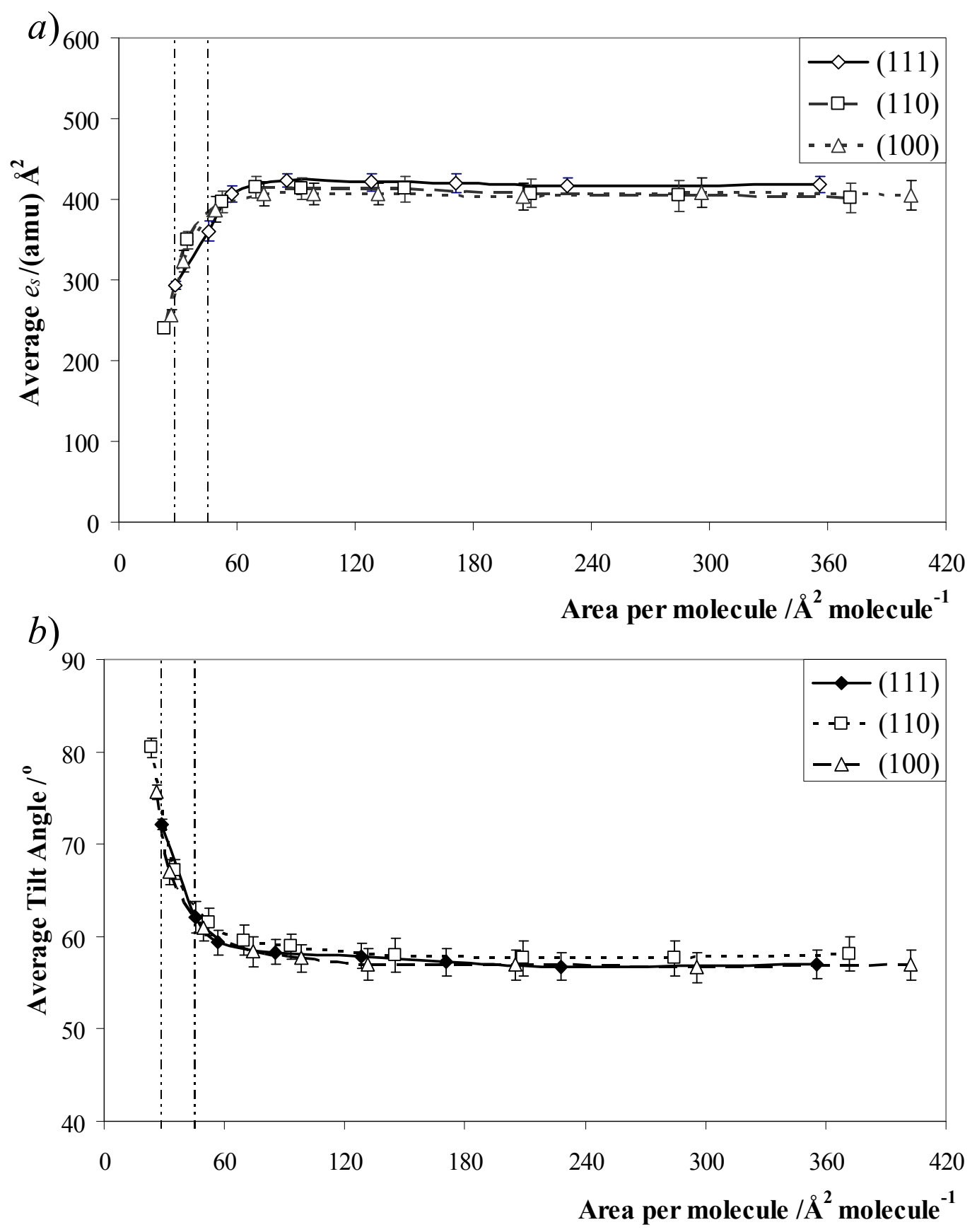

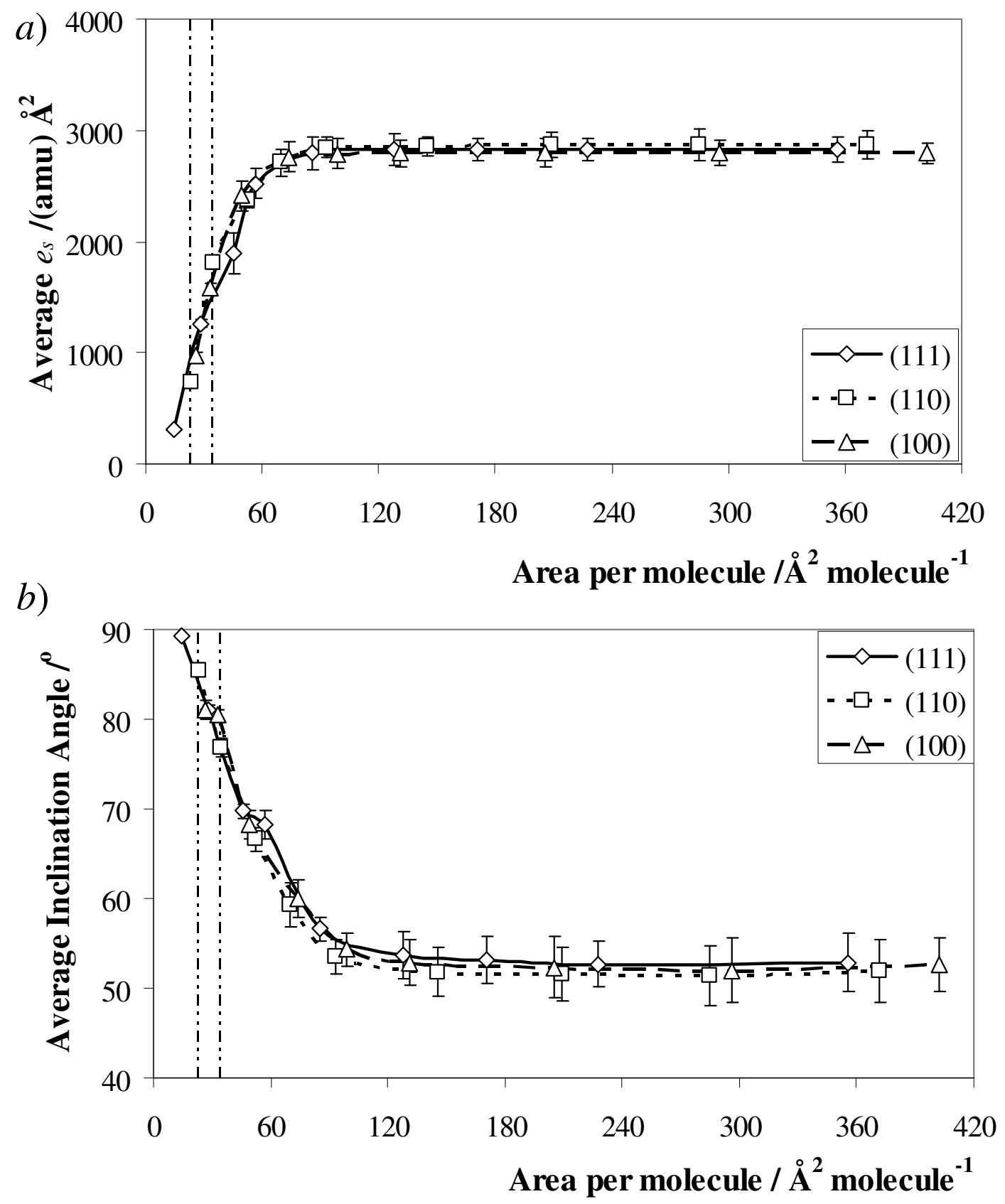


\title{
Simulation of the structure of organosilane film coatings
}

\author{
Robert A.Hayes ${ }^{\mathrm{a}}$, Graeme W. Watson ${ }^{\mathrm{b}}$ and David J. Willock ${ }^{\mathrm{a}}$. \\ a. School of Chemistry, Main Building, Cardiff University, Cardiff, CF10 3AT \\ b. School of Chemistry, University of Dublin, Trinity College, Dublin 2. \\ * Author to whom correspondence should be addressed.
}

\begin{abstract}
Organosilane compounds are easily coated onto metal and oxide substrates to form protective coatings. In this contribution we consider the intrinsic stability of these films using the surfaces of iron to define 2-D arrangements of the molecules within a film. Molecular dynamics simulation is used to analyse the packing energy and structure of the resulting films for two example organosilane molecules differing in chain length. The longer chain is found to form structures with higher absolute values of the packing energy.
\end{abstract}

\section{Introduction}

The coating of steel and other metals is important in the control and prevention of corrosion. The use of organosilanes in this area provides a more environmentally acceptable approach than the traditional chromium electroplating process which requires both high energy input and the disposal of toxic waste. However the integrity of the films produced by organosilane coatings is still a matter of concern and active research. Davis and Watts ${ }^{1}$ have published a combined study using XPS and ToFSIMS to explore the organisation of methoxysilane molecules on iron. They found that under the correct conditions a uniform silane layer of thickness $1.7 \mathrm{~nm}$ could be deposited. They also found that for short chain silanes the layer structure was complex, and far less ordered than for longer chain molecules.

These surface coatings are generally names for the methoxy silane molecules from which they are derived. We will consider two cases; 3glycidoxyeicosyltrimethoxysilane 3-GEMS and 3-glycidoxyepropyltrimethoxysilane 3-GPMS. In the coating solution the methoxy functional groups at the Si terminus of these molecules become hydroxylated. The silanes are then bound to a metal surface through a hydrolysis reaction with surface hydroxyl groups to give Fe-O-Si chemical bonds to the substrate material. ${ }^{2}$ An example surface bound structure for 3-GPMS is shown in figure 1 with a surface bond represented by a single Fe atom. Figure 1 also gives the forcefield potential types to be discussed in the methodology section. The main features of these molecules are the $-\mathrm{Si}(\mathrm{OH})_{2} \mathrm{O}$ - group which provides the anchor 
point to the surface and at the opposite terminus an epoxide functionality which can chemically react with secondary coatings such as paint layers. These two features are joined by a hydrocarbon chain, in the 3-GPMS example shown in figure 1 this is a propyl linkage with 3 carbon atoms, in 3-GEMS this is extended to 20 carbon atoms. Studying these two examples allows effect of the chain length on the structure of the coating to be considered.

Earlier molecular dynamics work by Kinloch and co-workers ${ }^{3}$ considered organosilanes adsorbed onto a series of oxide surfaces $\left(\alpha-\mathrm{Al}_{2} \mathrm{O}_{3}\right.$, amorphous- $\mathrm{Al}_{2} \mathrm{O}_{3}$, and $\mathrm{Fe}_{2} \mathrm{O}_{3}$ ) and was focused on direct comparison with the experimental data presented by the same group. ${ }^{4}$ In particular the film density was estimated from the experimental results. In this contribution we will investigate the effect of film density on the structure of the coating. In addition the interaction energy between silane molecules as a function of silane coverage allows an intrinsic optimal film density to be identified. It is likely that in the conditions used to deposit coatings the surface morphology and heterogeneity will also influence the surface density obtained. ${ }^{5}$

\section{Methodology}

The organosilane molecules in these simulations were represented using two, three and four body intra-molecular potentials along with atomic charges and van der Waals interactions taken from the consistent valence forcefield (CVFF) ${ }^{6}$. The atom types assigned are given in figure 1 , atom charges were assigned using the standard bond increments from the forcefield parameter set. Parameterisation of metallic Fe in such a scheme has not been carried out. However the goal of this study is to assess the film packing and so it was assumed that the silane headgroups are bonded to surface $\mathrm{Fe}$ atoms which act as anchor points fixing the $z$-coordinate of the oxygen atom bonded to the surface. However at low silane surface densities it was found that simply using the coordinated $\mathrm{Fe}$ atoms as constraints on the silane molecules was not sufficient because it is possible for chains to take on conformations in which the epoxide functionality is below the surface plane. To prevent this unphysical behavior each surface layer Fe centre was used as the centre of a pure repulsion potential of the form,

$U=\frac{a}{r^{12}}$ 
Taking $a=218600 \mathrm{~kJ} \mathrm{~mol}^{-1} \AA^{-1}$ gives an energy of $4 \mathrm{~kJ} \mathrm{~mol}^{-1}$ at the lattice $\mathrm{Fe}-\mathrm{Fe}$ spacing of $2.482 \AA$ so that the barrier introduced by the surface is of the order of $\mathrm{kT}$ at room temperature. This gives a barrier which maintains the integrity of the system without unduly affecting film structure. To apply the potential to interactions between the $\mathrm{Fe}$ atoms and the film the usual CVFF combining rules were applied.

All calculations used a simple cut-off, ${ }_{2}$ set at $8.0 \AA$, was employed for the van der Waals interaction potentials. Electrostatic contributions to the total energy were calculated using the Ewald method with a convergence criterion of $10^{-4} \mathrm{eV}$.

Deleted: method

Formatted: Superscript Three surfaces were considered based on $\mathrm{Fe}(111), \mathrm{Fe}(100), \mathrm{Fe}(110)$ these give two dimensional lattices with the geometries given in Table 1. The Fe sites on these lattices are used to locate the silane head groups and so the area per site and sites per $\mathrm{cm}^{2}$ give an indication of the effective film density at a coverage of 1 silane per surface Fe.

\begin{tabular}{ccccc}
\hline Surface & $\begin{array}{c}a=b \\
/ \AA\end{array}$ & $\begin{array}{c}\gamma \\
/ \text { degrees }\end{array}$ & $\begin{array}{c}\text { Area per site } \\
/ \AA^{2}\end{array}$ & $\begin{array}{c}\text { Sites per } \mathrm{cm}^{2} \\
/ 10^{14}\end{array}$ \\
\hline $\mathrm{Fe}(111)$ & 4.054 & 60.0 & 14.2 & 7 \\
$\mathrm{Fe}(100)$ & 2.866 & 90.0 & 8.2 & 12.2 \\
$\mathrm{Fe}(110)$ & 2.482 & 70.5 & 5.8 & 17.2 \\
\hline
\end{tabular}

Table 1 The geometry of the surfaces used to set the silane head group positions.

The starting structures for different surface densities of silanes were generated from a surface unit cell containing only one silane molecule per $\mathrm{Fe}$ atom, i.e. the full coverage surface density for each facet. To generate lower surface densities the full coverage cell was replicated to give an initial surface with enough sites to allow the appropriate number of silane molecules to be deleted. For example for $1 / 2$ and $1 / 4$ coverage the cell was doubled in the $a$ and $b$ lattice directions producing four independent silane molecules. To create a $1 / 2$ coverage cell two silane molecules were then removed while for $1 / 4$ coverage 3 molecules were deleted. Finally, the resulting basic cell was replicated to give a supercell containing multiple independent molecules at the required surface density. For basic cells containing a single silane molecule a $5 \times 5$ supercell was used to give 25 independent silane molecules. This was not possible for the half-coverage case for which a $4 \times 4$ supercell containing 32 independent molecules was produced and for the five-sixteenths coverage a $2 \times 2$ array containing 20 molecules was employed. A reference structure based on one molecule per twenty-five available surface sites for $\mathrm{Fe}(111)$, or one molecule per forty-nine available surface sites for $\mathrm{Fe}(110)$ and $\mathrm{Fe}(100)$ was also generated. The bound $\mathrm{Fe}$ 
atoms were assigned the same charge as the hydrogen atom they replaced $(0.119 e)$, to maintain charge neutrality, unbound Fe atoms were assigned a charge of zero. The FeO bond length (which was fixed) was set to $1.794 \AA$ (obtained from DFT level calculations of methylsilane chemisorption on an $\mathrm{Fe}(7,3)$ cluster $\left.^{7}\right)$.

All simulations were carried out using DLPOLY ${ }^{8}$ with a three dimensional simulation cell. The cell vector perpendicular to the Fe surface was chosen to ensure that the epoxide groups of the organosilane molecules in the fully extended conformation were at least $6 \AA$ from the repeating image Fe sites. The NVE ensemble was used throughout.

In our initial studies we were concerned that the MD simulation should be capable of generating a fully equilibrated film starting from the supercells in which the silane molecules are generated from periodic images of a single molecular structure. To develop the simulation protocol the $1 / 2$ coverage $\mathrm{Fe}(111)$ system was selected. Fe has the body centred cubic crystal structure (Space group $\operatorname{Im} \overline{3} \mathrm{~m})$ and so this surface has hexagonal symmetry. The $2 \times 2$ unit cell created in the first part of the cell construction contained 4 molecules of which 2 must be removed. Apparently there are two choices (figure $2 a$ and $b$ ) but these are actually equivalent by symmetry and so must give equivalent results in the MD runs. Indeed a radial distribution function (RDF) for the epoxide oxygen atoms $\left(\mathrm{O}_{3 \mathrm{e}}\right.$ defined in figure 1$)$ from a $200 \mathrm{ps}$ run at $300 \mathrm{~K}$ gave identical results as shown in figure $3 a$. However the calculated average energy of the two runs differed so that for the simulation based on the construction from figure $2 a$ the average energy was found to be $13 \mathrm{~kJ} \mathrm{~mol}^{-1}$ lower than that from a starting point based on figure $2 b$. Analysis of the RDF for the hydroxyl hydrogen atoms $\left(\mathrm{H}_{\mathrm{O}}\right.$ defined in figure 1), which are in the head group region of the silane molecules, also showed considerable differences between the two runs (figure $3 b$ ). The distribution generated for the higher energy configuration (from figure $2 b$ ) shows three well defined peaks suggesting a high degree of order in this section of the film. In contrast the simulation starting from the configuration from figure $2 a$ shows a broad peak and shoulder.

Although the two constructions are symmetry equivalent, when the shape of the organosilane molecule head group is taken into account the surface symmetry will be broken. During the MD run correct sampling of the silane configurations should reintroduce the symmetry equivalence of the structures. Figure 4 shows the arrangement 
of the silane head groups in snapshots of the trajectories after 200 ps of simulation. Figure $4 b$ confirms that this part of the molecule has maintained an ordered structure for the figure $2 b$ starting point whereas starting from figure $2 a$ disorder is introduced into the orientation of the head groups (figure $4 a$ ). The disordered surface pattern indicates that the symmetry of the surface will have been correctly sampled and gives the lower energy per molecule. These initial results indicated that a starting point using the construction in figure $2 b$ was unable to access the phase space found during the run starting from the figure $2 a$ point and so a simulation at $300 \mathrm{~K}$ is found to be non-ergodic.

To proceed the expected symmetry equivalence was used as a test for the simulation protocol and an annealing step was introduced with parameters chosen to give identical results from the two starting points. The final conditions used were as follows: Simulations were initially run for $100 \mathrm{ps}$ (at a reduced timestep of $0.01 \mathrm{fs}$ ) before a further $100 \mathrm{ps}$ run (at the usual timestep of $1 \mathrm{fs}$ ) at $300 \mathrm{~K}$ to remove any initial high forces introduced during the construction process. This was followed by a $100 \mathrm{ps}$ annealing run at $1700 \mathrm{~K}$ which was found to break down the head group ordering found initially for half coverage structures based on figure $2 b$. The final run of 300 ps was made up of 100 ps equilibration and 200 ps production all at a simulation temperature of $300 \mathrm{~K}$. All data for the MD simulations is collected in this final 200 ps run. Following this procedure equivalent results are obtained from either of the starting points illustrated in figure 2 .

\section{Results and Discussion}

The packing energy was defined as the difference between the average MD energy per silane molecule at a given surface density and that for the high dilutions of our reference structures. Hence the packing energy is the energy gained on forming a film from well separated, surface bound silane molecules. This packing energy has been calculated for 3-GPMS and 3-GEMS on the three lowest index surfaces of iron: $\mathrm{Fe}(100), \mathrm{Fe}(110)$ and $\mathrm{Fe}(111)$.

The calculated packing energy for 3-GPMS on each of the three Fe surface lattices is shown as a function of the area per molecule in figure 5. In all three cases as film density increases from the reference states at high area per molecule the interaction energy decreases smoothly. This indicates that there is a driving force for film 
formation through favourable interactions between organosilane molecules at intermediate separations. The $\mathrm{Fe}(111)$ surface shows a minimum in the interaction energy of around $-24 \mathrm{~kJ} \mathrm{~mol}^{-1}$ between $28 \AA^{2}$ per molecule and $46 \AA^{2}$ per molecule which corresponds to half occupation and $5 / 16^{\text {th }}$ occupation of the $\mathrm{Fe}$ sites respectively. The latter structure was obtained by inserting one additional molecule for every four in the quarter occupation simulation cell. The half coverage simulation was discussed in the methodology section where we showed that the head group sections of the organosilane layer are quite tightly packed at this surface coverage. However the organosilane head groups are quite bulky compared to the alkyl chain and epoxide sections and so it appears that dense films will be stabilised by interchain van der Waals forces.

A deeper minimum was found based on the $\mathrm{Fe}(110)$ surface with a calculated interaction energy of $-35 \mathrm{~kJ} \mathrm{~mol}^{-1}$ at $23.2 \AA^{2}$ per molecule. This corresponds to a quarter of the available surface sites being populated, however the $\mathrm{Fe}(110)$ surface contains more surface sites per unit area than does the $\mathrm{Fe}(111)$ and so we find a higher packing density on $\mathrm{Fe}(110)$ than on $\mathrm{Fe}(111)$.

The most favourable 3-GPMS interaction energy was found on the $\mathrm{Fe}(100)$ surface which has a regular square array of adsorption sites. At a surface area per molecule of $32.9 \AA^{2}$ per molecule we calculate an interaction energy of $-39 \mathrm{~kJ} \mathrm{~mol}^{-1}$. This, again, corresponds to one in four surface sites having a 3-GPMS molecule.

Figure 6 shows the calculated packing energy as a function of the area per molecule for the longer chain 3-GEMS organosilane on the three Fe surfaces. For the Fe(111) surface a minimum of $-72 \mathrm{~kJ} \mathrm{~mol}^{-1}$ is found at a packing of $28 \AA^{2}$ per molecule. This is in a similar position to that seen for 3-GPMS but the 3-GEMS minimum is much more sharply defined. The other two surfaces give more negative packing energies at similar densities to that found on $\mathrm{Fe}(111)$. The lowest packing energy is found for the $\mathrm{Fe}(110)$ surface with a value of $-101 \mathrm{~kJ} \mathrm{~mol}^{-1}$ at $23 \AA^{2}$ per molecule. We were unable to obtain results for higher densities on this surface since the MD simulations became unstable, indicating a large positive packing energy.

In general the optimal packing densities of the longer chain 3-GEMS organosilanes give packing energies that are more than twice that of the corresponding 3-GPMS films. This suggests that the increased van der Waals interaction per chain leads to intrinsically more stable coatings. 


\section{Film Structure}

A snapshot from the molecular dynamics run of 3-GPMS at $28 \AA^{2}$ per molecule is shown in figure 7. This is close to the optimal packing density obtained from the packing energy calculations. In this example it is clear that the chains are in an extended conformation roughly perpendicular to the surface. Importantly this places the epoxide functional groups at the vacuum interface so that they are available to bind with additional coatings such as paint layers.

To quantify the structure of the films we looked for numerical measures with which to describe the features discussed above that could be averaged across the $200 \mathrm{ps}$ molecular dynamics trajectories from the production phase of the calculations. We base our approach on the moments of inertia of molecules in the simulation. For a single molecule the moment of inertia matrix, $I_{M}$, is easily calculated from the atomic co-ordinates,

$$
I_{M}=\left(\begin{array}{ccc}
\sum_{i \in M} m_{i}\left(\left|r_{i}\right|^{2}-r_{i x}^{2}\right) & \sum_{i \in M} m_{i} r_{i x} r_{i y} & \sum_{i \in M} m_{i} r_{i x} r_{i z} \\
\sum_{i \in M} m_{i} r_{i x} r_{i y} & \sum_{i \in M} m_{i}\left(\left|r_{i}\right|^{2}-r_{i y}^{2}\right) & \sum_{i \in M} m_{i} r_{i y} r_{i z} \\
\sum_{i \in M} m_{i} r_{i x} r_{i z} & \sum_{i \in M} m_{i} r_{i y} r_{i z} & \sum_{i \in M} m_{i}\left(\left|r_{i}\right|^{2}-r_{i z}^{2}\right)
\end{array}\right)
$$

where $m_{i}$ is the mass of the $i^{\text {th }}$ atom in molecule $M$ and $r_{i}$ the vector between the $i^{\text {th }}$ atom and the centre of mass of the molecule with components $\left(r_{i x}, r_{i y}, r_{i z}\right)$. In the calculation of $I_{M}$ from the molecular dynamics trajectories the anchoring Fe sites were ignored.

Diagonalisation of the $I_{M}$ matrix yields three eigenvalues which correspond to the scalar moments of inertia for rotation of the molecule around the corresponding set of three perpendicular axes defined by the eigenvectors of $I_{M}$. If the eigenvalues are ranked in order, $e_{s}, e_{m}, e_{l}$, (small, medium and large), the eigenvector corresponding to $e_{s}$ will give the axis for which rotation is most facile. For molecules such as the organosilanes considered here this gives an unambiguous way to define the molecular axis direction and the extent of elongation of the molecule. The molecular axis can be taken from the eigenvector for $e_{s}$ because this defines a vector which corresponds to the long axis of the mass distribution in the molecule as shown in figure $8 a$. The extent of elongation of the molecules can be quantified by comparing the magnitude of $e_{s}$ between molecules of the same chemical formula. In general elongated molecules (figure $8 a$ ) will have smaller values of $e_{s}$ than molecules in conformations 
which are more compact since in the latter case the mass of the molecule will be distributed more widely from the axis (figure $8 b$ ). Using the eigenvector corresponding to $e_{s}$ also allows us to define a tilt angle for a molecule in the organosilane films as the angle made with the plane of the surface.

The value of $e_{s}$ and its corresponding eigenvector was calculated for each molecule in the simulation and for each of the 2500 frames saved to the trajectory file. The average value for 3-GPMS as a function of area per molecule in the film is shown in figure $9 a$ with error bars derived from the standard deviation for each data point. At low area per molecule the organosilanes are effectively isolated and we find a value of $e_{s}$ of just over $400 \mathrm{amu} \AA^{2}$. As the area per molecule is reduced this value remains constant until around $80 \AA^{2}$ molecule $^{-1}$ at which point a smooth decrease in $e_{s}$ is observed. So as the film density increases the molecules are adopting more elongated conformations consistent with closer packing. The optimal packing densities are indicated on figure $9 a$ and correspond to average $e_{s}$ values in the range 300-360 amu $\AA^{2}$. The surface facet controlling the geometry of the film does not appear to influence the molecular conformation to the same extent as the packing energy.

The average tilt angle for 3-GPMS as a function of area per molecule is given in figure $9 b$. At low coverages a tilt angle of around $58^{\circ}$ is observed and this increases to between 63 and $72^{\circ}$ in the optimal packing region. This means that as the molecules pack more closely together they are also orientated closer to the vertical to the surface plane. For both the average value of $e_{s}$ and the tilt angle the size of the error bars can be seen to reduce as the film density increases, indicating that inter-molecular interactions restrict the range of motion of the organosilanes.

Figure 10 shows the moment of inertia and tilt angle data for 3-GEMS on the three $\mathrm{Fe}$ surfaces as a function of the area per molecule in the film. The higher molecular mass of the longer molecule gives larger values of $e_{s}$ than were seen for the 3-GPMS example and a value of just under $3000 \mathrm{amu} \AA^{2}$ is observed at low film densities. This value is constant with coverage above $100 \AA^{2}$ molecule $^{-1}$, but below this value a decrease with increasing film density is again observed. At the optimal film densities we find $e_{s}$ is between 1000 and $1500 \mathrm{amu} \AA^{2}$, indicating that the effect of packing on the elongation of the molecule is more pronounced that for 3-GPMS. This probably arises from the greater conformational flexibility for the free $\mathrm{C} 20$ chain. The tilt angle dependence on surface coverage is shown in figure $10 b$. The isolated molecules in this case show a tilt angle of around $52^{\circ}$ with a standard deviation range of $5^{\circ}$. This 
standard deviation is larger than observed for 3-GPMS, again consistent with a larger number of accessible conformations for the longer chain. At the optimal packing density the tilt angle increases to between $79^{\circ}$ and $84^{\circ}$, so that the molecules are closer to being perpendicular to the surface plane than was observed for 3-GPMS. In addition the error bars are significantly reduced compared to the isolated 3-GEMS results indicative of inter-molecular interactions restricting the motion of the chains. For both 3-GPMS and 3-GEMS the particular facet of Fe employed has very little effect on the moment of inertia data obtained.

\section{Conclusions}

The interactions between the organosilane molecules favours dense packed films rather than isolated molecules on all three surfaces studied. The surface geometry of the Fe sites influences the optimal packing that can be achieved for the organosilane overlayer so that, for both 3-GPMS and 3-GEMS, $\mathrm{Fe}(110)$ and $\mathrm{Fe}(100)$ give films with more favourable interaction energies than does $\mathrm{Fe}(111)$.

The chain length has a significant affect on the calculated packing energies with 3GEMS having values more than twice those of the corresponding 3-GPMS films. In addition the 3-GEMS minima are more tightly defined. Analysis of the structure of the films using the molecular moments of inertia show that at the optimal packing densities the molecules are in extended conformations with the molecular orientation placing the epoxide group toward the vacuum interface.

Experimentally it has been found that the structure of the coating is more uniform for the longer chains. These simulations have concentrated on the intrinsic stability of the organosilane films with the surface sites present simply as a regular lattice of anchor points. In a real film the interaction with the surface would also be significant. However, our results suggest that the interactions between organosilane molecules increase with chain length and should always favour dense ordered films. The influence of the surface would be expected to remain constant for any given chain length and so it is likely that order is seen for 3-GEMS due to the greater influence of the intra-film interactions considered here.

\section{Acknowledgements}

We are grateful to CORUS for the award of a PhD studentship to RAH.

Figure Captions 


\section{References}

1. S. J. Davis and J. F. Watts, Int. J. Adhes. Adhes. 16, 5 (1996).

2. E. P. Plueddemann, H. A. Clark, L. E. Nelson and K. R. Hoffmann, Mod. Plast. 39, 136 (1962).

3. P. M. Hobbs and A. J. Kinloch, J. Adhes. 66, 203 (1997).

4. N. G. Cave and A. J. Kinloch, Polymer. 33, 1162 (1992).

5. J.Quinton, L.Thomsen and P.Dastoor, Surface and Interface Analysis, 25, 931, (1997).

6. P. Dauberosguthorpe, V. A. Roberts, D. J. Osguthorpe, J. Wolfe, M. Genest, and A. T. Hagler, Proteins Struct. Funct. Genet. 4, 31 (1988). 


\section{Page 21 of 21}

1

2

3

4

5

6

7

8

9

10

11

12

13

14

15

16

17

18

19

20

21

22

23

24

25

26

27

28

29

30

31

32

33

34

35

36

37

38

39

40

41

42

43

44

45

46

47

48

49

50

51

52

53

54

55

56

57

58

59

7. R.A.Hayes, G.W.Watson, D.J.Willock and H.Edwards, in "Foundations of Molecular Modeling and Simulations" (P.T. Cummings, P.R. Westmoreland, and B. Carnahan, eds.), AIChE Symposium Series No. 325, 97, 230, (2001).

8. W. Smith and T. Forester, J. Molec. Graphics, 14, 136, (1996). 